\title{
BMJ Open Use of heparins in patients with cancer: individual participant data meta- analysis of randomised trials study protocol
}

Holger J Schünemann, ${ }^{1,2}$ Matthew Ventresca, ${ }^{1,3}$ Mark Crowther, ${ }^{4}$ Matthias Briel, ${ }^{1,5}$ Qi Zhou, ${ }^{1}$ David Garcia, ${ }^{6}$ Gary Lyman, ${ }^{7,8}$ Simon Noble, ${ }^{9}$ Fergus Macbeth, ${ }^{10}$ Gareth Griffiths, ${ }^{10,11}$ Marcello DiNisio, ${ }^{12,13}$ Alfonso lorio, ${ }^{1,14}$ Joseph Beyene, ${ }^{1,15}$ Lawrance Mbuagbaw, ${ }^{16}$ Ignacio Neumann, ${ }^{1,17}$ Nick Van Es, ${ }^{13}$ Melissa Brouwers, ${ }^{18}$ Jan Brozek, ${ }^{1}$ Gordon Guyatt, ${ }^{1}$ Mark Levine, ${ }^{1}$ Stephan Moll, ${ }^{19}$ Nancy Santesso, ${ }^{1}$ Michael Streiff, ${ }^{20}$ Tejan Baldeh, ${ }^{1}$ Ivan Florez, ${ }^{1,21}$ Ozlem Gurunlu Alma, ${ }^{22}$ Ziad Solh, ${ }^{23}$ Walter Ageno, ${ }^{24}$ Maura Marcucci, ${ }^{25}$ George Bozas, ${ }^{26}$ Gilbert Zulian, ${ }^{27}$ Anthony Maraveyas, ${ }^{28}$ Bernard Lebeau, ${ }^{29}$ Harry Buller, ${ }^{13}$ Jessica Evans, ${ }^{10}$ Robert McBane, ${ }^{30}$ Suzanne Bleker, ${ }^{13}$ Uwe Pelzer, ${ }^{31}$ Elie A Akl, ${ }^{1,32}$ and the IPDMA heparin use in cancer patients research group

To cite: Schünemann HJ, Ventresca M, Crowther M, et al. Use of heparins in patients with cancer: individual participant data meta-analysis of randomised trials study protocol. BMJ Open 2016;6:e010569. doi:10.1136/bmjopen-2015010569

- Prepublication history and additional material is available. To view please visit the journal (http://dx.doi.org/ 10.1136/bmjopen-2015010569).

Received 9 December 2015 Accepted 10 December 2015

CrossMark

For numbered affiliations see end of article.

Correspondence to Dr Holger Schünemann; schuneh@mcmaster.ca

\section{ABSTRACT}

Introduction: Parenteral anticoagulants may improve outcomes in patients with cancer by reducing risk of venous thromboembolic disease and through a direct antitumour effect. Study-level systematic reviews indicate a reduction in venous thromboembolism and provide moderate confidence that a small survival benefit exists. It remains unclear if any patient subgroups experience potential benefits.

Methods and analysis: First, we will perform a comprehensive systematic search of MEDLINE, EMBASE and The Cochrane Library, hand search scientific conference abstracts and check clinical trials registries for randomised control trials of participants with solid cancers who are administered parenteral anticoagulants. We anticipate identifying at least 15 trials, exceeding 9000 participants. Second, we will perform an individual participant data metaanalysis to explore the magnitude of survival benefit and address whether subgroups of patients are more likely to benefit from parenteral anticoagulants. All analyses will follow the intention-to-treat principle. For our primary outcome, mortality, we will use multivariable hierarchical models with patient-level variables as fixed effects and a categorical trial variable as a random effect. We will adjust analysis for important prognostic characteristics. To investigate whether intervention effects vary by predefined subgroups of patients, we will test interaction terms in the statistical model.

Furthermore, we will develop a risk-prediction model for venous thromboembolism, with a focus on control patients of randomised trials.

Ethics and dissemination: Aside from maintaining participant anonymity, there are no major ethical concerns. This will be the first individual participant

\section{Strengths and limitations of this study}

- In addition to our extensive search and robust analysis, the strength of our study relates to the possibility of identifying patient-level effect modifiers and exploring the survival benefit suggested by our study level meta-analysis.

- This will be the first IPDMA to address the health effects of heparin on patients with cancer and to provide important background for decision makers in this field. Our results will likely receive attention in the next editions of several guidelines given the participation of several investigators in guideline development groups (eg, the American College of Chest Physicians, Cancer Care Ontario, American Society of Hematology, American Society of Clinical Oncology and National Comprehensive Cancer Network)

- A number of knowledge users will be directly involved in the project on various occasions. They will take ownership through the involvement with all stages of the project and coauthoring of the publications resulting from this knowledge synthesis.

- The American Hematological Society guideline panel has already been involved in the generation of the questions to be answered by this synthesis. We believe that this offers an outstanding opportunity for dissemination.

- The guideline developers will use the results of this synthesis to inform their guideline recommendations and practice. Several of the knowledge users will disseminate the results through presentations at clinical rounds as well as national and international conferences.

- Weaknesses of our study are in the risk of not obtaining data from all studies that will be identified. 
data meta-analysis addressing heparin use among patients with cancer and will directly influence recommendations in clinical practice guidelines. Major cancer guideline development organisations will use eventual results to inform their guideline recommendations. Several knowledge users will disseminate results through presentations at clinical rounds as well as national and international conferences. We will prepare an evidence brief and facilitate dialogue to engage policymakers and stakeholders in acting on findings.

Trial registration number: PROSPERO CRD42013003526.

\section{INTRODUCTION}

Cancer is a leading cause of death worldwide. In Canada, approximately 145000 new cases are diagnosed annually. ${ }^{1}$ The risk of venous thromboembolic complications is elevated in patients with cancer. ${ }^{2} 3$ The annual risk of suffering a venous thromboembolic event (VTE), estimated from control groups of randomised trials in patients with solid cancer, is $4-5 \% .{ }^{4}$ Patients who experience VTE frequently require hospital admission and/or prolonged anticoagulant therapy. VTE is also associated with impairments in function of day-to-day life and pain, in addition to increased costs. ${ }^{5}$

Heparin and other LMWHs, such as fondaparinux and danaparoid, do not have intrinsic anticoagulant activity but potentiate antithrombin III, which inhibits activated coagulation agents. These agents constitute indirect anticoagulants as their activity is mediated by plasma cofactors. ${ }^{6}$ Heparin and its low-molecular weight derivatives are not absorbed orally and must be administered parenterally by intravenous infusion or subcutaneous injections. ${ }^{7}$ Researchers have hypothesised that heparin may improve outcomes in patients with cancer through an antitumour effect in addition to its antithrombotic effect. ${ }^{8}$ This antitumour activity of heparin, mechanistically, includes the inhibition of cell-cell interaction by blocking cell-adhesion molecules (selectins), the inhibition of extracellularmatrix protease heparanase and the inhibition of angiogenesis. ${ }^{9}$ In a 1992 clinical trial comparing nadroparin, a $\mathrm{LMWH}$, to unfractionated heparin (UFH) in patients with proven deep venous thrombosis (DVT), nadroparin unexpectedly reduced mortality in the subgroup of patients with cancer. ${ }^{10}$ However, anticoagulants may increase the risk for bleeding and is likely higher in patients with cancer. Heparins are also known to cause heparin-induced thrombocytopaenia. ${ }^{11}$

We recently updated our study-level systematic review evaluating the role of parenteral anticoagulants in patients with cancer who have no other indication for anticoagulant therapy. ${ }^{412}$ We identified a number of randomised trials that, in the most recent iteration of our update, suggested a survival benefit and a large reduction in VTE. The results of the systematic review were used by the American College of Chest Physicians (ACCP) antithrombotic guideline panel in its ninth iteration of the guidelines. ${ }^{2}$ However, the members of this highly influential guideline panel made a conditional recommendation against the use of heparin because they were uncertain as to whether or not the intervention reduced mortality. Since then, new trials have been published and we have updated our study-level systematic review (see table 1 for the summary of findings table and online supplementary appendix 1 for the detailed evidence profile).

There are several key questions that remain unanswered by this study-level meta-analysis. First, the analysis of mortality remains inconclusive (the CI for the relative risk included the value of 1.0), but survival analysis suggested a statistically significant mortality benefit. Unfortunately, this cannot be further investigated without IPD. Second, given the broad inclusion criteria in the randomised controlled trials (RCTs), it is unclear whether the effects are restricted to subgroups of patients defined by the type (ie, primary tumour site) or stage of cancer (eg, local vs metastatic). ${ }^{13}$ Third, there is uncertainty about the appropriate duration of treatment and whether or not there is an interaction with the administration of chemotherapy and hormone therapy (ie, the coadministration of chemotherapy or hormone therapy may potentiate the beneficial effects of parenteral anticoagulants). Fourth, there is uncertainty whether heparin has a direct antitumour effect. IPDMA might be able to shed more light on this issue if survival is prolonged independently of the occurrence of VTE. Fifth, the baseline risk for VTEs and the risk of bleeding are unknown in many of these patients and it is possible that patient data from these trials can be used to develop risk-prediction models.

To develop the best recommendations for patient care and to make socially responsible decisions, guideline panels and others developing recommendations must be informed by the best possible summary of the evidence. Study-level meta-analysis does not allow for the evaluation of modifying factors and for survival analysis based on prognostic factors of individual patients. An IPDMA can help resolve these issues.

The results of this synthesis will directly inform the recommendations of the next update of several clinical practice guidelines that in turn will benefit clinicians and patients with cancer. Furthermore, the results of this project will inform decisions about the allocation of healthcare resources.

\section{OBJECTIVES}

The primary objective of this IPDMA of RCTs is to assess patient-important benefits and patient-important harms of parenteral anticoagulants in patients with cancer who have no other indication for anticoagulation. The use of IPD will allow us to place greater confidence on effect estimates and identify specific subgroups that are more likely to benefit.

We will focus on the following unanswered clinical questions: 
Table 1 Summary of findings for study-level meta-analysis of parenteral anticoagulants in cancer

\begin{tabular}{|c|c|c|c|c|c|}
\hline \multirow[b]{2}{*}{ Outcome† } & \multirow[b]{2}{*}{$\begin{array}{l}\text { Relative effect } \\
(95 \% \mathrm{Cl})\end{array}$} & \multicolumn{3}{|c|}{ Estimated absolute effects at 1 year } & \multirow[b]{2}{*}{$\begin{array}{l}\text { Certainty of } \\
\text { the effect }\end{array}$} \\
\hline & & $\begin{array}{l}\text { Without } \\
\text { LMWH } \\
\text { (per 1000) }\end{array}$ & $\begin{array}{l}\text { With LMWH } \\
\text { (per 1000) }\end{array}$ & $\begin{array}{l}\text { Difference } \\
\text { (per 1000) }\end{array}$ & \\
\hline Death & RR 0.94 (0.88 to 1.00$)$ & 501 & 471 & $\begin{array}{l}30 \text { fewer deaths } \\
\text { (60 to } 0 \text { fewer) }\end{array}$ & $\begin{array}{l}\oplus \oplus \bigcirc \\
\text { Moderate due to } \\
\text { imprecision }\end{array}$ \\
\hline $\begin{array}{l}\text { Symptomatic venous } \\
\text { thromboembolism }\end{array}$ & RR 0.57 (0.40 to 0.81$)$ & 46 & 26 & $\begin{array}{l}20 \text { fewer events } \\
\text { ( } 9 \text { to } 27 \text { fewer) }\end{array}$ & $\begin{array}{l}\oplus \oplus \oplus \oplus \\
\text { High }\end{array}$ \\
\hline Major bleeding & RR 1.06 (0.71 to 1.57$)$ & 16 & 17 & $\begin{array}{l}1 \text { more major bleed (from } 5 \\
\text { fewer to } 9 \text { more) }\end{array}$ & $\begin{array}{l}\oplus \oplus \oplus \bigcirc \\
\text { Moderate due to } \\
\text { imprecision }\end{array}$ \\
\hline Minor bleeding & RR 1.18 (0.89 to 1.55$)$ & 27 & 32 & $\begin{array}{l}5 \text { more minor bleeds (from } \\
3 \text { fewer to } 15 \text { more) }\end{array}$ & $\begin{array}{l}\oplus \oplus \oplus \oplus \\
\text { High }\end{array}$ \\
\hline
\end{tabular}

1. What is the impact on survival of parenteral anticoagulants administered to patients with cancer with no therapeutic or prophylactic indication for anticoagulation?

2. Which subgroups of patients with cancer are more likely to benefit than be harmed from anticoagulation?

3. If there is a survival benefit from the use of parenteral anticoagulation in patients with cancer, is this survival benefit a result of an antitumour effect?

Secondary objectives include seeking answers to the following questions:

1. Can biomarkers such as $\mathrm{C}$ reactive protein, D-dimers and P-selectin be used to monitor or diagnose disease?

2. Can risk-prediction models to foresee the occurrence of VTE be developed from patient data of included trials?

3. What is the performance of the Khorana score and extended Khorana score for predicting VTE?

\section{METHODS/DESIGNS}

This systematic review will be conducted according to standards of the Cochrane Collaboration. ${ }^{14}$ Our protocol is currently registered in the International Prospective Register for Systematic Reviews (PROSPERO), CRD42013003526. Our systematic review will adhere to the Preferred Reporting Items for Systematic Reviews and Meta-Analyses (PRISMA) guidelines for reporting systematic reviews. ${ }^{15}$

\section{Inclusion/exclusion criteria}

1. Types of studies: we will consider RCTs that provide IPD.

2. Types of participants: patients with solid cancers with no indication for prophylactic anticoagulation (eg, for acute illness, for central venous line placement, perioperative) or for therapeutic anticoagulation (eg, for the treatment of DVT or PE). These are typically patients with solid cancers undergoing chemotherapy and/or radiotherapy in the outpatient setting.

3. Types of intervention:

- Experimental intervention-parenteral anticoagulants such as UHF, LMWH and fondaparinux.

- Comparator intervention-placebo or no intervention.

- We will also consider studies comparing different parenteral anticoagulants. The protocol should have planned to provide all other cointerventions (eg, chemotherapy) similarly.

\section{Literature search}

We will update our 2011 search of the following electronic bibliographic databases: ${ }^{12}$ MEDLINE, EMBASE, The Cochrane Library (including Cochrane Central Register of Controlled Trials/CENTRAL, Clinical Trials, DARE and NHS EED). A professional librarian in collaboration with content, clinical and methodology experts will develop specific search strategies. There will be neither date nor language restrictions. We will also review references of identified studies and narrative review articles, and update our search of conference proceedings (conference proceedings of the American Society of Clinical Oncology and of the American Society of Hematology). We will also use the related article feature in PubMed to identify additional articles.

\section{Study selection}

The inclusion criteria for this review are identical to our previous study-level systematic review. We will attempt to obtain IPD for RCTs that were previously analysed ${ }^{12}$ and any new RCTs that we identify to be eligible. An overview of known RCT's we hope to include in eventual analysis is provided in table 2.

For the study selection process, two reviewers will independently assess titles and abstracts of all identified citations for potential eligibility based on predefined criteria mentioned above. We will retrieve the full-text 


\begin{tabular}{ll} 
Author year & Trial name \\
\hline Agnelli $2012^{16}$ & $\begin{array}{l}\text { Evaluation of Semuloparin sodium (AVE5026) in } \\
\text { the Prevention of venous thromboembolism in } \\
\text { patients with cancer undergoing chemotherapy } \\
\text { (SAVE-ONCO) }\end{array}$ \\
Agnelli 2009 & $\begin{array}{l}\text { PROphylaxis of ThromboEmbolism during } \\
\text { PR }\end{array}$
\end{tabular}

Agnelli $2009^{17}$ PROphylaxis of ThromboEmbolism during CHemoTherapy (PROTECHT)
Metastatic or locally chemotherapy with and without

low-molecular-weight heparin in small cell lung cancer

Altinbas

Haas $2005^{19}$

ow-molecular-weight heparin versus

Placebo for the prevention of venous

Thromboembolism in metastatic

breast cancer or stage III/IV lung cancer (TOPIC 1 and 2)

Kakkar $2004^{20}$

The Fragmin Advanced Malignancy Outcome Study (FAMOUS)

Advanced cancer Therapy (MALT)

\section{Type of cancer}

Patients with metastatic

or locally advanced

solid tumours advanced solid cancer receiving chemotherapy

SCLC

Advanced cancer

Dalteparin

374

Number of

patients

Anticoagulant randomised Outcomes

Semuloparin 3172

Nadroparin

1150

Metastatic breast or

Certoparin lung

Dalteparin

84

- Survival (12 months)

- VTE

- Major bleeding, minor bleeding

- Survival (4-12 months)

- Response to chemo (4 months)

- Thrombotic complications in patients with CVC

- Superficial thrombophlebitis of lower limbs

- Asymptomatic thromboembolic events

- Safety (bleeding, major, minor others, 4 months)

- All-cause mortality (12-24 months)

- Symptomatic DVT

Bleeding

- Survival (6 months)

- Major and minor bleeding

- Venous thromboembolism

- Symptomatic and asymptomatic DVT

- PE-Vena subclavia, vena jugularis

- Superficial thrombophlebitis

Symptomatic venous thromboembolism

- Any thrombotic event

- Skeletal events

- Mortality (12-24-36 months)

- Symptomatic venous thromboembolism

- (PE, DVT)

Major bleeding

- Minor bleeding

- Mortality (6-12-24 months)

- Major bleeding

- Clinically relevant non-major bleeding

- All clinically relevant bleeding (major and non-major combined) 


\begin{tabular}{|c|c|c|c|c|c|}
\hline Author year & Trial name & Type of cancer & Anticoagulant & $\begin{array}{l}\text { Number of } \\
\text { patients } \\
\text { randomised }\end{array}$ & Outcomes \\
\hline Lebeau $1994^{22}$ & $\begin{array}{l}\text { Subcutaneous heparin treatment } \\
\text { Increases survival in small cell } \\
\text { Lung cancer }\end{array}$ & SCLC & $\begin{array}{l}\text { Unfractionated } \\
\text { heparin }\end{array}$ & 277 & $\begin{array}{l}\text { Survival (12-24-36 months) } \\
\text { Bleeding }\end{array}$ \\
\hline $\begin{array}{l}\text { Lecumberri } \\
2010^{23}\end{array}$ & $\begin{array}{l}\text { Adjuvant BEmiparin in small cell Lung carcinoma } \\
\text { (ABEL) }\end{array}$ & SCLC & Bemiparin & 26 & $\begin{array}{l}\text { Mortality (18 months) } \\
\text { - Bleeding } \\
\text { - Tumour progression }\end{array}$ \\
\hline $\begin{array}{l}\text { Maraveyas } \\
2011^{24}\end{array}$ & $\begin{array}{l}\text { A phase II randomised study of } \\
\text { chemoanticoagulation (gemcitabine-dalteparin) vs } \\
\text { chemotherapy alone (gemcitabine) for locally } \\
\text { advanced and metastatic pancreatic } \\
\text { adenocarcinoma (FRAGEM) }\end{array}$ & Pancreatic cancer & Dalteparin & 123 & $\begin{array}{l}\text { VTE } \\
\text { Survival } \\
\text { Toxicity } \\
\text { Time to disease progression } \\
\text { - Serological markers of } \\
\text { thromboangiogenisis }\end{array}$ \\
\hline Macbeth $^{25}$ & $\begin{array}{l}\text { A randomised phase III clinical trial investigating } \\
\text { the effect of FRAGMin added to standard therapy } \\
\text { in patients with lung cancer (FRAGMATIC) }\end{array}$ & Lung cancer & Dalteparin & 2202 & $\begin{array}{l}\text { Survival } \\
\text { Serious adverse events } \\
\text { Toxicity } \\
\text { - Quality of life } \\
\text { Cost-effectiveness }\end{array}$ \\
\hline $\begin{array}{l}\text { Pelzer et al } \\
2008^{26}\end{array}$ & $\begin{array}{l}\text { A prospective, randomised trial of simultaneous } \\
\text { pancreatic cancer treatment with enoxaparin and } \\
\text { ChemoTherapy (PROSPECT) }\end{array}$ & $\begin{array}{l}\text { Advanced pancreatic } \\
\text { cancer on } \\
\text { chemotherapy }\end{array}$ & Enoxaparin & 312 & $\begin{array}{l}\text { Overall survival } \\
\text { Symptomatic VTE } \\
\text { Major bleeding } \\
\text { Time to progression }\end{array}$ \\
\hline Perry $2010^{27}$ & $\begin{array}{l}\text { A trial of dalteparin low molecular weight heparin } \\
\text { for primary prophylaxis of venous } \\
\text { thromboembolism in brain tumour patients } \\
\text { (PRODIGE) }\end{array}$ & Malignant glioma & Dalterparin & 186 & $\begin{array}{l}\text { Primary outcomes: symptomatic } \\
\text { DVT or PE. } \\
\text { Secondary outcomes bleeding } \\
\text { (major and all bleeding) } \\
\text { Quality of life } \\
\text { - Cognition assessments } \\
\text { Death }\end{array}$ \\
\hline $\begin{array}{l}\text { Sideras } \\
2006^{28}\end{array}$ & $\begin{array}{l}\text { Low-molecular-weight heparin in patients with } \\
\text { advanced cancer: a phase } 3 \text { clinical trial }\end{array}$ & Advanced cancer & Dalteparin & 137 & $\begin{array}{l}\text { Mortality (12 and } 24 \text { months) } \\
\text { - Symptomatic VTE } \\
\text { - Major/minor bleeding }\end{array}$ \\
\hline $\begin{array}{l}\text { Van Doormaal } \\
2011^{29}\end{array}$ & $\begin{array}{l}\text { Improving with nadroparin the Prognosis in } \\
\text { advanced cancer treatment (INPACT) }\end{array}$ & $\begin{array}{l}\text { NSCLC } \\
\text { Prostate cancer } \\
\text { Pancreatic cancer }\end{array}$ & Nadroparin & 503 & $\begin{array}{l}\text { Mortality } \\
\text { Disease progression } \\
\text { - Major/minor bleeding } \\
\text { - Venous thromboembolism (PE, DVT) }\end{array}$ \\
\hline
\end{tabular}




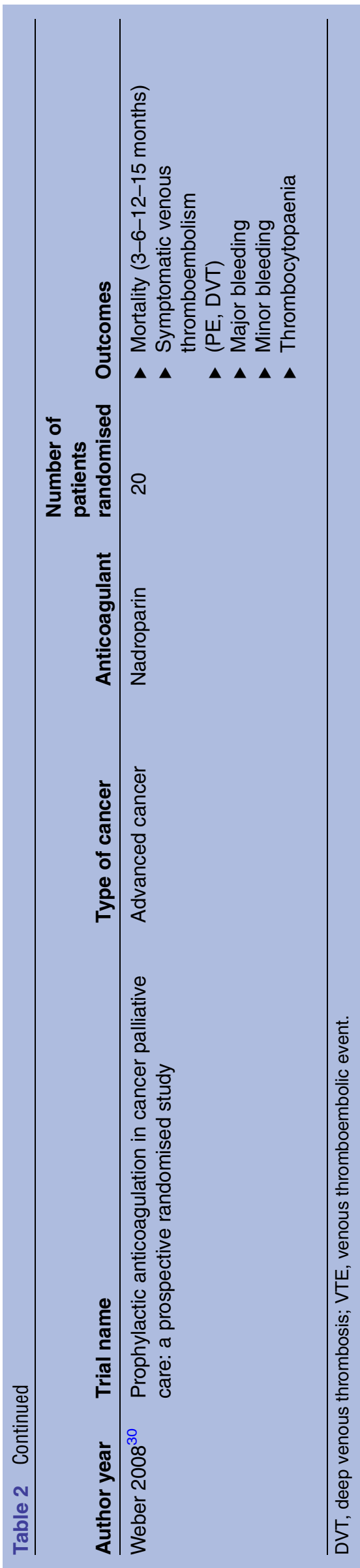

articles of all citations deemed eligible by at least one reviewer. Two reviewers will then screen the full texts for eligibility using a standardised pilot tested form with explicit inclusion and exclusion criteria. Decisions will be compared and agreement will be measured using the к statistic. ${ }^{31}$ Disagreements will be resolved by consensus and, when needed, with the help of a third reviewer. We will record reasons for exclusion.

We will contact authors of all included trials by email, fax or telephone, and invite them to share their data. Specifically, we will request the study protocol, a copy of the case report forms and the corresponding dataset with all patient identifiers (eg, names) removed. In addition to the study protocol and complete analysis plans, a detailed list of all variables of interest will be provided to contacted trialists in order to maintain analytical transparency, to avoid data driven analysis and to encourage apprehensive authors to share relevant trial data, excluding sensitive or unnecessary information from being disseminated. Data should include important demographic variables that are required for the analysis and, if possible, biomarker information.

\section{Assessment of risk of bias and overall quality of evidence}

Two review authors will assess, in duplicate and independently, the risk of bias in the included trials, using a standardised form. They will compare their results and resolve disagreements by discussion or with the help of a third reviewer. We will use the following criteria to assess the risk of bias: Allocation concealment; blinding of participants, healthcare providers, data collectors, outcome adjudicators, and data analysts; proportion of participants with available data; and stopping early for benefit. We will assess selective outcome and other reporting bias by comparing the list of outcomes in published protocols and in the methods section to the outcomes reported in the published paper. We will assess the risk of bias and overall quality of evidence in the study-level meta-analysis as an initial approach. We will generate an inverted funnel plot for each comparison to check for possible publication bias. We will assess statistical heterogeneity by calculating the $\mathrm{Q} \chi^{2}$ and its $\mathrm{p}$ value, and the $\mathrm{I}^{2}$. We will record and report the sponsorship of the studies (whether sponsored by a forprofit or not-for-profit organisation or government agency), and assess financial and intellectual conflicts of interest.

We will prepare GRADE evidence profiles to summarise the information about the treatment effects following the GRADE approach ${ }^{17}$ to provide support for decision makers, using our GRADE profiler software. ${ }^{18}$ For this purpose, we will expand the existing GRADE methodological framework to incorporate assessing the quality of evidence coming from IPDMA. For that, we will assess publication bias and heterogeneity by using study-level identifiers. We will conduct this assessment for the relative estimates of effect as well as baseline risk estimates in various patient subgroups. 


\section{Datasets and data extraction}

The primary purpose of the project is to conduct an IPDMA; if any investigators are unable or unwilling to provide us with their database we will compare, in a secondary analysis, the results using study-level data, in order to include all trials. We will request trial protocols, final trial reports and unedited databases including all randomised patients. Two review authors will independently extract individual participant data and aggregate level data in duplicate using standardised prepiloted data extraction forms. They will compare their results and resolve disagreements by discussion or with the help of a third reviewer. Data of interest include demographic, intervention and outcome data.

Trialists will be assured that their data will be treated confidentially. All data sets received will be stored in a secure location in their original formats. Prior to data extraction, we will create a list of baseline variables (eg, age, sex, date of cancer diagnosis, site of primary cancer) and outcome variables (eg, mortality, bleeding, DVT, PE) to be used in the IPDMA. We will then try to identify each of these variables in each of the trial data sets. During this stage, we will check definitions of variables in the individual trials and, if necessary, create our own definitions to ensure commonality of terms between trials. We will then adapt or recalculate some of the original variables to ensure consistency of definitions. We will request and extract data about all outcomes of interest necessary to conduct analyses following the intention-to-treat (ITT) principle.

All data sets will be worked on separately in order to standardise variable names and value labels across trials. Data will initially be entered into an Excel database and converted to SAS for analysis. Finally, we will collate data sets and assign a trial variable with a code for each trial. For each study, relevant descriptive data of all enrolled patients will be presented, including demographic data (eg, age and gender), underlying diagnosis (type of cancer and stage), other baseline clinical and laboratory data, including defining criteria for the stage of their disease, other blood markers of inflammation (eg, D-dimer and P-selectin), results of diagnostic tests and prognostic scores or comorbidities.

We will match data after recoding of variables into common variables. The percentage of missing data in analyses relevant variables will be calculated and Little's method will be used to assess the missing completely at random (MCAR) assumption in the variable with at least $5 \%$ of missing data. In case the MCAR is violated, we will further examine the MAR pattern by comparing the outcome in the variable with and without missing values. Thus, we will extract data on:

\section{Participants}

1. Number of patients randomised to each treatment arm

2. Number of patients followed-up in each treatment arm
3. Number of patients who withdrew from treatment in each treatment arm

4. Number of patients who discontinued treatment in each treatment arm

5. Population characteristics (age, sex, duration, co-morbidities, and type and stage of cancer, co-interventions)

6. Inflammatory markers (P-selectin, C reactive protein, D-dimer)

Interventions

1. Type of parenteral anticoagulant studied

2. Dosage of treatment

3. Duration of treatment

4. Inpatient versus outpatient treatment

Outcomes

1. All-cause mortality; prespecified at 12 months, 24 months and over the duration of the trial

2. Overall and symptomatic venous thromboembolism (DVT and/or PE): DVT events must be diagnosed using an objective diagnostic test such as: venography, or compression ultrasound. PE events have to be diagnosed using an objective diagnostic test such as: pulmonary perfusion/ventilation scans, CT, pulmonary angiography or autopsy

3. Health-related quality of life: has to be measured using a validated tool

4. Major bleeding: we will accept the authors' definitions of major bleeding

5. Minor bleeding: we will accept the authors' definitions of minor bleeding

6. Thrombocytopaenia

\section{Data analysis}

We plan to use SAS V.9.3 (Cary, North Carolina, USA) or a newer version of this software to analyse data. All analyses will follow the ITT principle with all patients analysed according to the arm to which they were randomised. A consistency check of the raw data from all trials will be performed. In addition, to ensure that the data provided correspond to the reported results, we will reproduce and recalculate the primary outcomes of the trials and several randomly chosen baseline data.

For the variable that has missing data, we will use a multiple imputation method to impute the missing data. We will use the imputed data sets as the main study datasets for the statistical analyses. We will summarise the data in mean or median, depending on the distribution, for the continuous outcomes (eg, quality of life) together with SD or IQR. We will use count and percentage to describe the categorical data. We will express the intervention effects in OR for the dichotomous outcomes, HR for the time to the event outcomes and mean difference for the continuous outcomes along with the 95\% CIs.

In the regression analyses, we will use multilevel models $^{32-34}$ to incorporate the data at trial level and patient level. For the dichotomous outcomes, we will be using a logistic mixed model. Since a considerable number of trials $(>10)$ may be included, we plan to use 
a random-effects model, representing the joint distribution of the treatment effect and trial with a bivariate normal distribution (ie, using model (3) from Turner et $\left.a l^{4}\right)$. Assuming that the trials included in analysis are a random sample from a larger pool of trials, a random-effects model may be inappropriate. Therefore, in addition to random-effects models, fixed-effects models will also be completed for primary outcomes. A Cox proportional hazard regression model will be used for time to event outcomes. The heterogeneity among the trials will be modelled with frailty random effect. For the continuous outcomes, we will be using mixed regression model with random intercept to account the variability among the trials. The adjusted variables of comorbidities and age will be included as fixed-effects in the model. Residuals will be examined to assess the appropriateness of the model assumptions. We will fit corresponding models for our prespecified secondary outcomes. We will also report the anticipated absolute effects (eg, risk difference or number-needed-to-treat), based on the estimated OR and HR, and three assumed baseline risks and median or mean difference in survival. All data will be summarised in a GRADE evidence profile.

In addition, we will perform several prespecified sensitivity analyses to investigate differences in pooled effect estimates related to conduct or methods. We will also perform subgroup analyses to investigate differences in pooled effect estimates related to different patient subgroups. We will test whether there is a differential intervention effect among the various subgroups with an interaction-test, which is preferred to separate subgroup group-specific analyses. ${ }^{35} 36$

Subgroup analyses will be performed for the following variables:

1. Underlying diagnosis of included patients (type and stage of cancer): We hypothesise that patients with earlier stage cancer have greater benefit than those with later stage cancer. We also hypothesise, based on our prior analysis, that patients with lung or pancreatic cancer experience a greater benefit from anticoagulant therapy. ${ }^{13}$

2. Concomitant treatment (ie, chemotherapy compared with no chemotherapy): In patients treated with chemotherapy we anticipate larger effects of heparin.

Sensitivity analyses:

We have prespecified the following sensitivity analyses:

1. Compare the results of a study-level meta-analysis including studies for which we will not receive original datasets with the main IPDMA results. We will then explore if the study-level data differ after excluding the studies for which we do not receive original data sets.

2. Higher risk of bias in the original study may be associated with a greater effect. We will conduct sensitivity analysis by comparing results of trials judged at high risk of bias compared to those judged at low risk of bias (those that are judged as not suffering from any of the methodological limitations listed in the risk of bias assessment above).

For the secondary objective of developing a riskprediction model for VTE in patients with cancer we will perform logistic regression or Cox proportional hazard regression and competing risk analysis (until first occurrence of outcome), stratified by cancer type in patients not receiving therapy, using the studies for which we will have received IPD sets. The candidate predictors will be age, gender, cancer stage, chemotherapy, use of erythropoietin stimulating agents, statin use, the biochemical markers (P-selectin, $\mathrm{C}$ reactive protein and D-dimer), smoking status, obesity (body mass index), platelet count, leucocyte count and haemoglobin level if available (12). The dependent variable will be the composite of symptomatic lower extremity DVT and PE. Using manual stepwise backward selection, we will determine the final model, retaining the predictors with the strongest statistical effect and those classically considered risk factors (eg, specific chemotherapies). To adjust for overfitting, regression coefficients will be shrinked as needed. The regression coefficients will be used to calculate individual annual risks and corresponding 95\% CIs. Bootstrapping procedures will be performed for internal validation of the performance of the new rule.

In parallel, we will perform an external validation of the Khorana score ${ }^{37}$ and extended Khorana score, ${ }^{38}$ using the individual data of patients not receiving LMWH. We will recalculate individual risk for patients at baseline (expected, E) using the Korana score and the extended model including D-dimer and soluble P-selectin concentration. We will then assess the incidence of VTE (observed, O), for each score on the Khorana or extended Khorana score. We will perform net classification analysis and offset regression to verify the need for recalibration, and proceed to recalibrate if necessary. We will also dichotomise the Khorana score and extended Khorana score (cut-off $\geq 3$ points) to recalculate the sensitivity, specificity, negative predictive value and positive predictive value on the validation set.

\section{DISCUSSION}

This will be the first IPDMA to address the health effects of heparin on patients with cancer and to provide important background for decision makers in this field. Our results will likely receive attention in the next editions of several guidelines given the participation of several investigators in guideline development groups (eg, the American Society of Hematology, Cancer Care Ontario, American Society of Clinical Oncology and National Comprehensive Cancer Network). A number of knowledge users will be directly involved in the project on various occasions. They will take ownership through the involvement with all stages of the project and coauthoring of the publications resulting from this knowledge synthesis. Our next guidelines will be conducted 
with the American Society of Hematology (ASH) and the VTE in Cancer has formulated questions to be answered by this synthesis. We believe that this offers an outstanding opportunity for dissemination. The guideline developers will use the results of this synthesis to inform their guideline recommendations and practice. Several of the knowledge users will disseminate the results through presentations at clinical rounds as well as national and international conferences. Weaknesses of our study are in the risk of not obtaining data from all studies that will be identified. In addition to our extensive search and robust analysis, the strength of our study relates to the possibility of identifying patient-level effect modifiers and exploring the survival benefit suggested by our study-level meta-analysis.

\section{Author affiliations}

${ }^{1}$ Department of Clinical Epidemiology and Biostatistics, McMaster University, Hamilton, Ontario, Canada

${ }^{2}$ Department of Medicine, McMaster University, Hamilton, Ontario, Canada

${ }^{3}$ Department of Community Health Studies, Brock University, St Catharines, Ontario, Canada

${ }^{4}$ St Joseph's Hospital, and Department of Pathology and Molecular Medicine, McMaster University, Hamilton, Ontario, Canada

${ }^{5}$ Basel Institute for Clinical Epidemiology and Biostatistics, University Hospital Basel $\mathrm{CH}$, Basel, Switzerland

${ }^{6}$ University of Washington School of Medicine, Seattle, Washington, USA

${ }^{7}$ Hutchinson Institute for Cancer Outcomes Research, Fred Hutchinson Cancer Research Center, Seattle, Washington, USA

${ }^{8}$ Department of Medicine, University of Washington School of Medicine, Seattle, Washington, USA

${ }^{9}$ Marie Curie Palliative Care Research Centre, Cardiff University, Wales, UK

${ }^{10}$ Wales Cancer Trials Unit, School of Medicine, Cardiff University, Wales, UK

${ }^{11}$ Faculty of Medicine, University of Southampton, Southampton General

Hospital, Southampton, UK

${ }^{12}$ Department of Medical, Oral and Biotechnological Sciences, University "G

D'Annunzio" of Chieti-Pescara, Chieti, Italy

${ }^{13}$ Department of Vascular Medicine, Academic Medical Center, Amsterdam, The Netherlands

${ }^{14}$ Division of Hematology, Department of Medicine, Hamilton, Ontario, Canada

${ }^{15}$ The Hospital for Sick Children, Toronto, Ontario, Canada

${ }^{16}$ Biostatistics Unit, Father Sean O'Sullivan Research Centre, St Joseph's Healthcare, Hamilton, Ontario, Canada

${ }^{17}$ Department of Internal Medicine, School of Medicine, Pontificia Universidad

Católica de Chile, Santiago, Chile

${ }^{18}$ Department of Oncology, Escarpment Cancer Research Institute, McMaster University, Hamilton, Ontario, Canada

${ }^{19}$ Division of Hematology-Oncology, Department of Medicine, University of North Carolina School of Medicine, Chapel Hill, North Carolina, USA

${ }^{20}$ Department of Hematology, The Johns Hopkins University School of Medicine, Baltimore, Maryland, USA

${ }^{21}$ Department of Paediatrics, Universidad de Antioquia, Medellin, Colombia

${ }^{22}$ Department of Statistics, Mugla S tk Kocman Unv, Mugla, Turkey

${ }^{23}$ Division of Hematology/Oncology, Department of Pediatrics, McMaster

University, Hamilton, Ontario, Canada

${ }^{24}$ Department of Clinical and Experimental Medicine, University of Insubria, Varese, Italy

${ }^{25}$ Department of Clinical Sciences and Community Health, University of Milan

\& Geriatrics, Fondazione-IRCCS Ca' Granda Ospedale Maggiore Policlinico,

Milan, Italy

${ }^{26}$ Academic Department of Medical Oncology, Castle Hill Hospital, Cottingham, Hull and East Yorkshire Hospitals NHS Trust, UK

${ }^{27}$ Department of Readaptation and Palliative Medicine, Geneva University Hospitals, Switzerland

${ }^{28}$ Division of Cancer-Hull York Medical School, University of Hull, Hull, UK
${ }^{29}$ Service de Pneumologie, Hôpital Saint-Antoine, Assistance PubliqueHôpitaux de Paris, Université Pierre et Marie Curie, Paris, France

${ }^{30}$ Cardiology and Hematology Departments, Mayo Clinic, Rochester,

Minnesota, USA

${ }^{31}$ Division of Hematology, Oncology and Tumor Immunology, Medical Department, Charité Comprehensive Cancer Center, Charité Medical University, Berlin, Germany

${ }^{32}$ Department of Internal Medicine, American University of Beirut, Lebanon

Twitter Follow Holger Schünemann at @Schunemann_mac and Ivan Florez at @ivand_florez

Acknowledgements This study was supported by the Canadian Institutes of Health Research (http://www.cihr-irsc.gc.ca), grant KRS 126594, and registered in Prospero (CRD42013003526).

Contributors HS conceived of the study and obtained funding. The concept and design of this study were generated primarily by HS, EAA, MB, MC, JB and GL. A Knowledge Synthesis grant proposal submitted by HS, EAA, MB, MB, JB, JB, MC, GG, Lavis, GL and NS to The Canadian Institutes for Health Research funded this project. The study protocol was primarily drafted by $\mathrm{HS}, \mathrm{EAA}, \mathrm{MCr}$, JB and $\mathrm{GG}$, however, all the authors provided feedback and offered insight regarding potential revisions. MV was responsible for providing administrative support.

Funding This project is funded by a Knowledge Synthesis grant awarded by the Canadian Institutes for Health Research (grant KRS 126594).

Competing interests DG has been a consultant or received research funding from Boehringer Ingelheim, Bristol-Myers Squibb, CSL Behring,

Daiichi-Sankyo, Janssen, Pfizer and Portola. SN is on the advisory boards for Leo Pharma, Pfizer, Bristol Meyers Squibb and Bayer. He is honoraria for Leo Pharma, Pfizer and Boheringer Ingelheim, and has received grants from Leo Pharma and Pfizer. GG has consulted Pfizer on trial design and has also received free drugs from Pfizer for cancer related trials under the UK National Cancer Research Institute. MD has received consulting fees from Bayer Health Care and Grifols. SM has received consulting fees from Portola. MS has received research funding from Portola and has consulted for Daiichi-Sankyo, Boehringer, Pfizer and Janssen Healthcare. AM is an advisory board honoraria for Leo Pharma and Bayer. WA has accepted consulting fees from Bayer, Boehringer Ingelheim, Pfizer, Bristol Meyers Squibb, Daiichi-Sankyo and Italfarmaco. WA has also received research support from Bayer. None of the other authors report any conflicts of interest.

Ethics approval McMaster University Research Ethics Board.

Provenance and peer review Not commissioned; peer reviewed for ethical and funding approval prior to submission.

Data sharing statement Requests for additional details regarding the study protocol may be made by contacting the corresponding author, Dr Holger Schünemann (holger.schunemann@mcmaster.ca).

Open Access This is an Open Access article distributed in accordance with the Creative Commons Attribution Non Commercial (CC BY-NC 4.0) license, which permits others to distribute, remix, adapt, build upon this work noncommercially, and license their derivative works on different terms, provided the original work is properly cited and the use is non-commercial. See: http:// creativecommons.org/licenses/by-nc/4.0/

\section{REFERENCES}

1. Canadian Cancer Society's Advisory Committee on Cancer Statistics. Canadian Cancer Statistics 2015. Toronto, ON: Canadian Cancer Society; 2015.

2. Kahn SR, Lim W, Dunn AS, et al. Prevention of VTE in nonsurgical patients: antithrombotic therapy and prevention of thrombosis, 9th ed: American college of chest physicians evidence-based clinical practice guidelines. Chest 2012;141Suppl):e195S-226S.

3. Kucher N, Koo S, Quiroz R, et al. Electronic alerts to prevent venous thromboembolism among hospitalized patients. N Engl J Med 2005;352:969-77.

4. Akl EA, Kahale LA, Ballout RAet al. Parenteral anticoagulation in patients with cancer who have no therapeutic or prophylactic indication for anticoagulation. Cochrane Database Syst Rev 2014;12: CD006652. 
5. Anthony M. Nursing assessment of deep vein thrombosis. Medsurg Nurs 2013;22:95-8, 123

6. Hirsh J, Bauer K, Donati MB, et al. Parenteral anticoagulants: American college of chest physicians evidence-based clinical practice guidelines (8th Edition). Chest 2008;133(6 Suppl):141S-59S.

7. Hirsh J. Low molecular weight heparin. Thromb Haemost 1993;70:204-7.

8. Thodiyil P, Kakkar AK. Can low-molecular-weight heparins improve outcomes in patients with cancer? Cancer Treat Rev 2002;28:151-5.

9. Borsig L. Heparin as an inhibitor of cancer progression. Prog $\mathrm{Mol}$ Biol Transl Sci 2010;93:335-49.

10. Prandoni $\mathrm{P}$, Lensing AW, Buller HR, et al. Comparison of subcutaneous low molecular-weight heparin with intravenous standard heparin in proximal deep-vein thrombosis. Lancet 1992;339:441-5

11. Girolami B, Girolami A. Heparin-induced thrombocytopenia: a review. Semin Thromb Hemost 2006;32:803-9.

12. Akl EA, Schünemann HJ. Routine heparin for patients with cancer? One answer, more questions. N Engl J Med 2012;366:661-2

13. Lee AY. Thrombosis in cancer: an update on prevention, treatment, and survival benefits of anticoagulants. Hematology Am Soc Hematol Educ Program 2010;2010:144-9.

14. Higgins JPT, Green S, ed. Cochrane handbook for systematic reviews of interventions version 5.1.0 [updated March 2011]. The Cochrane Collaboration, 2011.

15. Stewart LA, Clarke M, Rovers $M$, et al. Preferred Reporting Items fo Systematic Review and Meta-Analyses of individual participant data: the PRISMA-IPD Statement. JAMA 2015;313:1657-65.

16. Agnelli G, George DJ, Kakkar AK, et al, SAVE-ONCO Investigators. Semuloparin for thromboprophylaxis in patients receiving chemotherapy for cancer. N Engl J Med 2012;366:601-9.

17. Agnelli G, Gussoni G, Bianchini $C$, et al. Nadroparin for the prevention of thromboembolic events in ambulatory patients with metastatic or locally advanced solid cancer receiving chemotherapy: a randomised, placebo-controlled, double-blind study. Lancet Oncol 2009;10:943-9.

18. Altinbas M, Coskun HS, Er O, et al. A randomized clinical trial of combination chemotherapy with and without low-molecular-weight heparin in small cell lung cancer. J Thromb Haemost 2004;2:1266-71.

19. Haas SK, Freund M, Heigener D, et al., TOPIC Investigators Low-molecular-weight heparin versus placebo for the prevention of venous thromboembolism in metastatic breast cancer or stage III/IV lung cancer. Clin Appl Thromb-Hem 2012;18:159-65.

20. Kakkar AK, Levine MN, Kadziola Z, et al. Low molecular weight heparin, therapy with dalteparin, and survival in advanced cancer: the fragmin advanced malignancy outcome study (FAMOUS). J Clin Oncol 2004;22:1944-8.

21. Klerk CPW, Smorenburg SM, Otten HM, et al. The effect of low molecular weight heparin on survival in patients with advanced malignancy. J Clin Oncol 2005;23:2130-5.
22. Lebeau B, Chastang C, Brechot JM, et al. Subcutaneous heparin treatment increases survival in small cell lung cancer. "Petites Cellules" Group. Cancer 1994;74:38-45.

23. Lecumberri R, Massuti B, Vivanco GL, et al. Adjuvant bemiparin in small cell lung cancer: results from the ABEL study. Thromb Res 2010;125:S163-S63.

24. Maraveyas A, Waters J, Roy R, et al. Gemcitabine versus gemcitabine plus dalteparin thromboprophylaxis in pancreatic cancer. Eur J Cancer 2012;48:1283-92.

25. Macbeth F, Noble S, Evans J, et al. Randomized Phase III Trial of Standard Therapy Plus Low Molecular Weight Heparin in Patients With Lung Cancer: FRAGMATIC Trial. J Clin Oncol 2016;34:488-94.

26. Pelzer U, Opitz B, Deutschinoff G, et al. Efficacy of Prophylactic Low-Molecular Weight Heparin for Ambulatory Patients With Advanced Pancreatic Cancer: Outcomes From the CONKO-004 Trial. J Clin Oncol 2015;33:2028-34.

27. Perry JR, Julian JA, Laperriere NJ, et al. PRODIGE: a randomized placebo-controlled trial of dalteparin low-molecular-weight heparin thromboprophylaxis in patients with newly diagnosed malignant glioma. J Thromb Haemost 2010;8:1959-65.

28. Sideras K, Schaefer PL, Okuno SH, et al. Low-molecular-weight heparin in patients with advanced cancer: a phase 3 clinical trial Mayo Clin Proc 2006;81:758-67.

29. van Doormaal FF, Di Nisio M, Otten HM, et al. Randomized trial of the effect of the low molecular weight heparin nadroparin on survival in patients with cancer. J Clin Oncol 2011;29:2071-6.

30. Weber C, Merminod T, Herrmann FR, et al. Prophylactic anti-coagulation in cancer palliative care: a prospective randomised study. Support Care Cancer 2008;16:847-52.

31. McGinn T, Wyer PC, Newman TB, et al. Tips for learners of evidence-based medicine: 3 . Measures of observer variability (kappa statistic). CMAJ 2004;171:1369-73.

32. Simmonds MC HJ, Stewart LA, Tierney JF, et al. Meta-analysis of individual patient data from randomized trials: a review of methods used in practice. Clin Trials 2005;2:209-17.

33. Thompson SG TR, Warn DE. Multilevel models for meta-analysis, and their application to absolute risk differences. Stat Methods Med Res 2001;10:375-92.

34. Turner RM OR, Yang M, Goldstein $\mathrm{H}$, et al. A multilevel model framework for meta-analysis of clinical trials with binary outcomes. Stat Med 2000;19:3417-32.

35. Assmann SF, Pocock SJ, Enos LE, et al. Subgroup analysis and other (mis) uses of baseline data in clinical trials. Lancet 2000;355:1064-9.

36. Brookes ST, Whitely E, Egger M, et al. Subgroup analyses in randomized trials: risks of subgroup-specific analyses; power and sample size for the interaction test. J Clin Epidemiol 2004;57:229-36.

37. Khorana AA, Kuderer NM, Culakova E, et al. Development and validation of a predictive model for chemotherapy-associated thrombosis. Blood 2008;111:4902-7.

38. Ay C, Dunkler D, Marosi C, et al. Prediction of venous thromboembolism in cancer patients. Blood 2010;116:5377-82. 